\title{
The value of left atrial strain and strain rate in predicting left atrial appendage stasis in patients with nonvalvular atrial fibrillation
}

\author{
Meng-Ruo Zhu, Man Wang, Xin-Xin Ma, Dong-Yan Zheng, Yue-Li Zhang
}

Department of Ultrasound in Medicine, Shanghai Jiao Tong University Affiliated Sixth People's Hospital, Shanghai Institute of Ultrasound in Medicine, Shanghai, China

\begin{abstract}
Background: This study aimed to investigate the value of left atrial (LA) strain and strain rate (S/SR) by transthoracic echocardiography (TTE) in predicting left atrial appendage (LAA) stasis, in order to find a way for LAA stasis screening which is easily performed in patients with nonvalvular atrial fibrillation (NVAF).

Methods: One hundred and thirty NVAF patients prepared for AF ablation were enrolled. TTE and transesophageal echocardiography (TEE) were performed in all patients. LA S/SR in each phase was analyzed off-line. LAA blood flow state and LAA function were assessed by using TEE.

Results: $L A S / S R$ s during atrial reservoir phase $\left(L A S_{r e s} / S R_{r e s}\right)$ were significantly negatively correlated with $L A A$ spontaneous echo contrast (SEC) grade $(r=-0.567$ and -0.520 , respectively; all $p<0.01)$, and positively correlated with LAA emptying fraction ( $r=0.602$ and 0.619 , respectively; all $p<0.01)$ and with LAA peak emptying flow velocity $(r=0.623$ and 0.642 , respectively; all $p<0.01)$. The multivariate logistic regression analysis showed $L A S_{\text {res }}$ to be the strongest independent predictor of $L A A$ stasis, followed by $L A$ volume index. $L A S_{\text {res }}<13 \%$ was recommended to predict $L A A$ stasis with sensitivity of $90 \%$ and specificity of $74 \%$.

Conclusions: $L A S_{\text {res }}$ by TTE can noninvasively predict LAA stasis and may be used as a screening tool to assist in the detection of LAA stasis in patients with NVAF. (Cardiol J 2018; 25, 1: 87-96)

Key words: strain, strain rate, left atrial, left atrial appendage stasis, nonvalvular atrial fibrillation, noninvasive
\end{abstract}

\section{Introduction}

Atrial fibrillation $(\mathrm{AF})$ increases the risk of thromboembolic events. It was reported that dominant source of embolism (over $90 \%$ ) is the left atrial (LA) appendage (LAA) in nonvalvular AF (NVAF) [1]. Although transesophageal echocardiography (TEE) is a superior mode of imaging in detecting thrombi in the LAA, the frequent use of this imaging technique is limited due to its semiinvasiveness [2]. So there is a need to find a noninvasive and applicable assisted screening method.
The primary mechanism of LA thrombus formation in patient with AF was thought to be blood stagnation induced by the lack of organized LA and LAA contraction. LAA function was reported to be correlated with LAA stasis [3]. Considering the structural integrity and functional consistency of LA and LAA, there should be a relation between LA function and LAA stasis. Two-dimensional (2D) speckle tracking echocardiography (STE) derived strain and strain rate imaging (S/SRI) has been confirmed to be a noninvasive and effective method in the quantification of LA mechanic function [4].

Address for correspondence: Yue-Li Zhang, MD, PhD, Department of Ultrasound in Medicine, Shanghai Jiao Tong University Affiliated Sixth People's Hospital, Shanghai Institute of Ultrasound in Medicine, 600 Yishan Road, Shanghai, 200233, China, e-mail: zhangyueli2002@163.com 
Table 1. Comparison of general clinical characteristics among three groups.

\begin{tabular}{|c|c|c|c|c|c|}
\hline & \multirow{2}{*}{$\begin{array}{l}\text { All patients } \\
(n=130)\end{array}$} & \multicolumn{3}{|c|}{ Groups } & \multirow[t]{2}{*}{$\mathbf{P}$} \\
\hline & & $1(n=81)$ & $2(n=28)$ & $3(n=21)$ & \\
\hline Age [years] & $63.5 \pm 9.2$ & $63.1 \pm 10.0$ & $65.8 \pm 6.5$ & $61.9 \pm 8.9$ & 0.279 \\
\hline Female gender [\%] & $51(39 \%)$ & $37(46 \%)$ & $10(36 \%)$ & $4(19 \%)$ & 0.076 \\
\hline $\mathrm{BSA}\left[\mathrm{m}^{2}\right]$ & $1.78 \pm 0.18$ & $1.86 \pm 0.20$ & $1.77 \pm 0.17$ & $1.76 \pm 0.18$ & 0.064 \\
\hline Heart rate [bpm] & $82 \pm 22$ & $77 \pm 20$ & $91 \pm 25^{*}$ & $90 \pm 20^{*}$ & 0.003 \\
\hline Persistent AF [\%] & $71(55 \%)$ & $29(36 \%)$ & $23(82 \%)^{*}$ & $19(90 \%)^{*}$ & 0.000 \\
\hline AF episode duration $>1$ year [\%] & $66(51 \%)$ & $40(49 \%)$ & $15(54 \%)$ & $11(52 \%)$ & 0.918 \\
\hline $\mathrm{CHA}_{2} \mathrm{DS}_{2}$-VASc & $1.98 \pm 1.20$ & $1.96 \pm 1.09$ & $1.96 \pm 1.09$ & $2.05 \pm 1.50$ & 0.987 \\
\hline Hypertension & $80(62 \%)$ & $48(59 \%)$ & $18(64 \%)$ & $14(67 \%)$ & 0.779 \\
\hline Diabetes mellitus & $21(16 \%)$ & $13(16 \%)$ & $4(14 \%)$ & $4(19 \%)$ & 0.904 \\
\hline Vascular disease & $6(5 \%)$ & $2(2 \%)$ & $2(7 \%)$ & $2(10 \%)$ & 0.301 \\
\hline Age $\geq 75$ years & $11(9 \%)$ & $7(9 \%)$ & $3(11 \%)$ & $1(5 \%)$ & 0.757 \\
\hline History of coronary artery disease & $14(11 \%)$ & $10(12 \%)$ & $3(11 \%)$ & $1(5 \%)$ & 0.607 \\
\hline Current smoking & $23(18 \%)$ & $14(18 \%)$ & $5(18 \%)$ & $4(19 \%)$ & 0.982 \\
\hline Hyperlipidemia & $18(14 \%)$ & $8(10 \%)$ & $5(18 \%)$ & $5(24 \%)$ & 0.202 \\
\hline eGFR $\left[\mathrm{mL} / \mathrm{min} / 1.73 \mathrm{~m}^{2}\right]$ & $71.3 \pm 18.3$ & $72.8 \pm 16.5$ & $71.9 \pm 17.3$ & $65.2 \pm 20.8$ & 0.144 \\
\hline \multicolumn{6}{|l|}{ Medications: } \\
\hline Warfarin & $54(42 \%)$ & $34(42 \%)$ & $11(39 \%)$ & $9(43 \%)$ & 0.961 \\
\hline INR & $1.51 \pm 0.66$ & $1.49 \pm 0.68$ & $1.52 \pm 0.59$ & $1.57 \pm 0.65$ & 0.294 \\
\hline
\end{tabular}

${ }^{*} \mathrm{p}<0.05$, versus group 1 ; data are expressed as mean \pm standard deviation or number (percentage); AF — atrial fibrillation; BSA — body surface area; eGFR — estimated glomerular filtration rate; INR — international normalized ratio

Thus, the possibility of LAA stasis being existent might be inferred from LA S/SR on transthoracic echocardiography (TTE). In this study, the value of LA S/SR in predicting LAA stasis was investigated in patients with NVAF.

\section{Methods}

\section{Study population}

One hundred and thirty-four patients with NVAF referred to our echo lab for TEE evaluation before AF ablation from February 2014 to April 2015 were recruited. NVAF was diagnosed following the 2014 AHA/ACC/HRS guideline [5]. Patients enrolled might also have following concomitant diseases: hypertension, diabetes mellitus, and coronary heart disease. The exclusion criteria were significant aortic or mitral valve disease (any degree of aortic/mitral valve stenosis or mitral/aortic regurgitation $>$ II), cardiomyopathy, any systemic disease such as significant liver disease, overt organ failure, neurologic disorders, malignant disease, hyperthyroidism, acute pulmonary disease or history of cardiac surgery. Warfarin was administered at the discretion of the physician in charge. The anticoagulation status was listed in Table 1.
This study was approved by the medical ethics committee of our hospital, and informed consent was obtained from all patients.

\section{Echocardiography}

All patients underwent TTE and TEE using a Philips iE33 ultrasound machine (Philips Medical Systems, Eindhoven, The Netherlands), with synchronous electrocardiogram (ECG) recording. All measurements were either given as the average values of 3 consecutive cardiac cycles in normal sinus rhythm (NSR) or the average values of 5 consecutive cardiac cycles during the AF episode.

\section{TTE}

A complete and standard TTE was performed using a Philips iE33, equipped with a S5-1 probe. All measurements and evaluations were performed according to the guidelines of American Society of Echocardiography [6]. The average heart rate was recorded. The left ventricular ejection fraction was measured using the modified Simpson's biplane method. Transmitral peak early diastolic filling velocity $\mathrm{E}$ was recorded by pulsed-wave Doppler at the tips of the mitral valve leaflets in an apical 4-chamber view and tissue Doppler imag- 
ing was applied in the pulsed-wave Doppler mode to record the mitral annulus peak early diastolic velocities (e') at the septal and lateral positions. The left ventricle (LV) filling index $\mathrm{E} / \mathrm{e}$ ' ratio was calculated using the average of two positions of the mitral annulus. LA volumes were calculated from the apical 4- and 2-chamber views of the LA using the biplane method of the discs. The maximum LA volume was indexed by dividing the body surface area to acquire the LA volume index (LAVI).

\section{Offline analyses - 2D STE S/SR}

Two-dimensional apical 4-chamber views acquired with at least 60 frames per second were digitized during 5 consecutive cardiac cycles in cine-loop format.

Views were imported to the $2 \mathrm{D}$ speckletracking workstation, TomTec-Image Arena 4.0 (2D Cardiac Performance Analysis; TomTec Imaging System, Munich, Germany). Each view was analyzed according to the following steps: using QRS onset of the ECG as the reference point, 5 cardiac cycles were selected. Next, the LA endocardial surface was traced manually by a point-and-click approach. An epicardial surface tracing was automatically generated by the system, creating a region of interest (ROI), which was manually adjusted to cover the full thickness of the LA wall. Before processing, a cine-loop preview feature visually confirmed that the internal line followed the LA endocardium throughout the 5 cardiac cycles. The software divided the ROI into 7 segments in each view and automatically calculated the average of 7 LA segments and generated average time-longitudinal S and SR curves. For each cardiac cycle, we identified the LA reservoir $\mathrm{S}\left(\mathrm{S}_{\mathrm{res}}\right)$ $=$ peak positive longitudinal $\mathrm{S}$ during ventricular systole - basic longitudinal S at the initial time of this cardiac cycle (corresponding to QRS onset of the ECG) from the average longitudinal S curve as an index for LA global reservoir function (Fig. 1). Peak positive longitudinal SR during the ventricular systole $\left(\mathrm{SR}_{\mathrm{res}}\right)$ and peak negative longitudinal $\mathrm{SR}\left(\mathrm{SR}_{\text {cond }}\right)$ during the ventricular early diastolic were identified from the average longitudinal SR curve, which were obtained to represent LA global reservoir and conduit function.

\section{TEE}

All subjects underwent TEE performed with a Philips iE33 (Philips Medical System, Eidhoven, The Netherlands), equipped with a S7-2 probe. With the subjects in the left lateral decubitus position, LAA was clearly visualized by turning the probe to the left and then flexing it to bring the LAA to the center of the imaging plane. Five consecutive cardiac cycles were acquired in cineloop format and stored for further analysis. LAA flow velocity was obtained by pulse-wave Doppler with the sample volume placed $1 \mathrm{~cm}$ away from the LAA orifice within the LAA cavity and Doppler signals were recorded in 5 to 10 consecutive cardiac cycles. The main parameters were observed and measured as follow:

1. LAA emptying function: The LAA area was measured by tracing a line from the top of the limbus of the left superior pulmonary vein along a straight line drawn to the aorta at the shortest distance of the base of the LAA and along the whole LAA endocardial border. The LAA maximal areas $\left(\mathrm{LAA}_{\max }\right)$ were measured just before the $\mathrm{P}$ wave (end of LAA diastole) and LAA minimal areas $\left(\mathrm{LAA}_{\min }\right)$ were measured at or just after the QRS complex (end of LAA systole) by planimetry when patients in NSR. In patients with AF, the $\mathrm{LAA}_{\max }$ and $\mathrm{LAA}_{\min }$ were visually determined by two observers independent of the ECG and measurements. The LAA emptying fraction (LAA-EF) was calculated by the method described by Pollick and Taylor as $\left(\left[\mathrm{LAA}_{\max }-\mathrm{LAA}_{\min }\right]\right.$ / $\left./ \mathrm{LAA}_{\max } \times 100 \%\right)$ [3].

2. LAA flow velocity profile: The LAA peak emptying flow velocities and filling flow velocities were measured.

3. The presence of SEC and thrombus in LA or LAA: The spontaneous echo contrast (SEC) was defined as dynamic, smoke-like echoes swirling slowly in a circular pattern within the LA or LAA cavity despite adjustment in the gain setting and graded from 0 to $4+$ according to the severity of SEC proposed by Fatkin et al. [7]. Organized thrombus was defined echocardiographically as a well-circumscribed, uniform-consistency, highly reflective mass adhering to the wall of the LA or LAA. When dense SEC (grade $3+$ or $4+$ ) was present and organized into a dynamic and gelatinous, but not solid or well-formed echodensity present throughout the cardiac cycle, sludge was reported [8]. Inter-observer differences in the grading of SEC and the presence of thrombus were resolved by consensus. As the sludge within LAA is an independent predictor of an increased risk for thromboembolism, whose rate is comparable with that related to LAA thrombus [9], LAA stasis was defined when sludge and thrombus were present in LAA. 


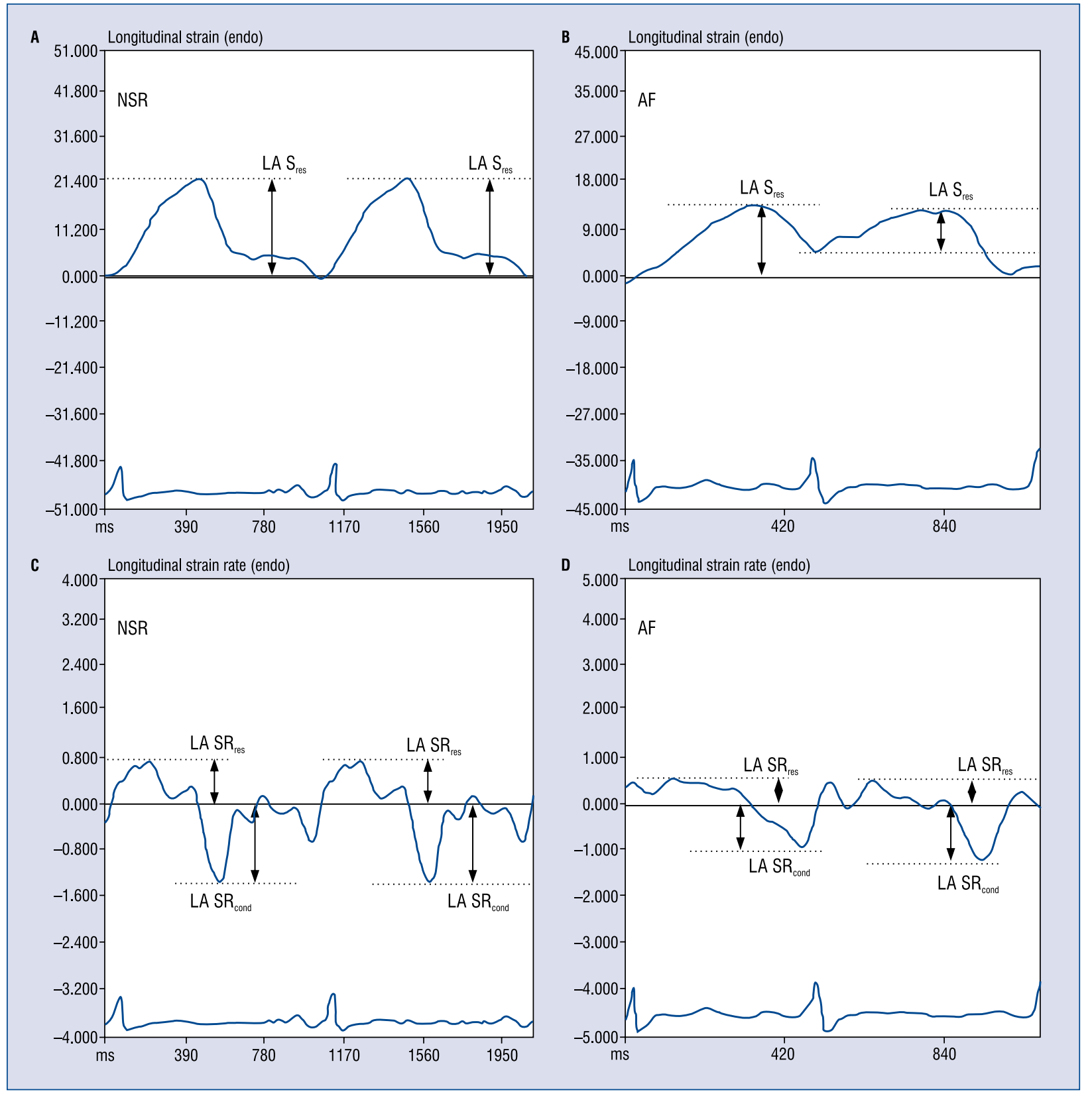

Figure 1. A-D. Left atrial (LA) strain and strain rate (S/SR) measurements in normal sinus rhythm (NSR) and atrial fibrillation $(A F) ; S_{\text {res }}$ - global longitudinal strain during atrial reservoir phase; $\mathrm{SR}_{\text {res }}$ - global longitudinal strain rate during atrial reservoir phase; $\mathrm{SR}_{\text {cond }}$ - global longitudinal strain rate during atrial conduit phase.

The study subjects were classified into three groups according to SEC grade: group 1, SEC grade 0 (none); group 2 , SEC grade $1+$ and $2+$ (slight to mild); group 3 , SEC grade $3+$ and $4+$ (moderate to severe), and thrombus.

\section{Intra-observer and inter-observer variability}

Twenty images randomly selected respectively from NSR and AF patients were analyzed by a second observer who was blinded to the values obtained by the first observer to assess the interobserver variability, and analyzed at a different time by the observer who was blinded to the results of the previous measurements to assess the intraobserver variability. Inter- and intra-observer reproducibility was assessed by calculating variability coefficients. Inter-observer variability coefficients were $4.4 \%, 4.1 \%$ and $-5.0 \%$ for $\mathrm{LA} \mathrm{S}_{\mathrm{res}}, \mathrm{SR}_{\mathrm{res}}$ and 
Table 2. Comparison of echocardiographic parameters among three groups.

\begin{tabular}{|c|c|c|c|c|c|}
\hline & \multirow{2}{*}{$\begin{array}{l}\text { All patients } \\
(n=130)\end{array}$} & \multicolumn{3}{|c|}{ Groups } & \multirow[t]{2}{*}{$\mathbf{P}$} \\
\hline & & $1(n=81)$ & $2(n=28)$ & $3(n=21)$ & \\
\hline \multicolumn{6}{|l|}{ TTE parameters } \\
\hline LV EF [\%] & $61.0 \pm 7.3$ & $61.3 \pm 6.9$ & $62.4 \pm 4.2$ & $57.8 \pm 10.5^{\#}$ & 0.303 \\
\hline E/e' ratio & $10.3 \pm 3.2$ & $10.2 \pm 3.4$ & $9.6 \pm 2.6$ & $11.2 \pm 3.2$ & 0.205 \\
\hline LAVI $\left[\mathrm{mL} / \mathrm{m}^{2}\right]$ & $37 \pm 13$ & $32 \pm 12$ & $44 \pm 11^{*}$ & $50 \pm 10^{* \#}$ & 0.000 \\
\hline \multicolumn{6}{|l|}{ TEE parameters } \\
\hline LAA area $\max _{\max }\left[\mathrm{cm}^{2}\right]$ & $5.9 \pm 2.1$ & $5.3 \pm 1.5$ & $6.3 \pm 1.8^{*}$ & $7.7 \pm 2.9^{* \#}$ & 0.000 \\
\hline LAA area $a_{\min }\left[\mathrm{cm}^{2}\right]$ & $3.9 \pm 2.1$ & $3.0 \pm 1.4$ & $4.6 \pm 1.7^{*}$ & $6.3 \pm 2.7^{* \#}$ & 0.000 \\
\hline LAA emptying fraction [\%] & $37.7 \pm 18.9$ & $45.9 \pm 17.3$ & $27.6 \pm 13.8^{*}$ & $19.5 \pm 10.1^{* *}$ & 0.000 \\
\hline LAA peak emptying flow velocity $[\mathrm{cm} / \mathrm{s}]$ & $49.1 \pm 28.1$ & $60.5 \pm 26.5$ & $38.6 \pm 21.2^{*}$ & $19.4 \pm 8.9^{* *}$ & 0.000 \\
\hline LAA peak filling flow velocity $[\mathrm{cm} / \mathrm{s}]$ & $51.1 \pm 23.7$ & $61.2 \pm 20.8$ & $44.1 \pm 16.5^{*}$ & $21.6 \pm 11.5^{* *}$ & 0.000 \\
\hline
\end{tabular}

* $\mathrm{p}<0.05$, versus group $1 ;{ }^{*} \mathrm{p}<0.05$, versus group 2; data are expressed as mean \pm standard deviation; E - early diastolic mitral inflow; $\mathrm{e}^{\prime}$ - early diastolic mitral annular tissue velocity; LAA — left atrial appendage; LAVI — left atrial maximal volume indexed to body mass area; LV EF - left ventricular ejection fraction; TEE — transesophageal echocardiography; TTE — transthoracic echocardiography

Table 3. Comparison of the left atrial strain and strain rate among three groups.

\begin{tabular}{lccccc}
\hline & All patients & \multicolumn{3}{c}{ Groups } & P \\
\cline { 3 - 5 } & $(\mathbf{n}=130)$ & $\mathbf{1}(\mathbf{n}=\mathbf{8 1})$ & $\mathbf{2}$ (n=28) & $\mathbf{3}$ (n= 21) \\
\hline LA S $_{\text {res }}[\%]$ & $20.1 \pm 11.9$ & $24.5 \pm 12.3$ & $15.1 \pm 6.7^{*}$ & $9.5 \pm 3.0^{* *}$ & 0.000 \\
LA SR $_{\text {res }}[1 / \mathrm{s}]$ & $0.91 \pm 0.40$ & $1.06 \pm 0.40$ & $0.77 \pm 0.29^{*}$ & $0.54 \pm 0.20^{* *}$ & 0.000 \\
LA SR $_{\text {cond }}[1 / \mathrm{s}]$ & $-1.10 \pm 0.43$ & $-1.17 \pm 0.47$ & $-1.10 \pm 0.36$ & $-0.83 \pm 0.27^{* \#}$ & 0.006 \\
\hline
\end{tabular}

${ }^{*} \mathrm{p}<0.05$, versus group 1 ; ${ }^{*} \mathrm{p}<0.05$, versus group 2; data are expressed as mean \pm standard deviation; LA — left atrial; SR $\mathrm{cond}_{\text {- }}$ global longitudinal strain rate during atrial conduit phase; $\mathrm{S}_{\text {res }}-$ global longitudinal strain during atrial reservoir phase; $\mathrm{SR}_{\text {res }}-\mathrm{global}_{\text {longitudinal strain }}$ rate during atrial reservoir phase

$\mathrm{SR}_{\text {cond }}$ in NSR patients, respectively. Corresponding intra-variability coefficients were $3.0 \%, 3.4 \%$ and $-3.3 \%$. In $\mathrm{AF}$ patients, inter-observer variability coefficients were $6.7 \%, 6.8 \%$ and $-7.0 \%$ for $\mathrm{LA}$ $\mathrm{S}_{\mathrm{res}}, \mathrm{SR}_{\mathrm{res}}$ and $\mathrm{SR}_{\text {cond }}$. Corresponding intra-variability coefficients were $5.7 \%, 5.4 \%$ and $-6.3 \%$, respectively.

\section{Statistical analysis}

Continuous data were presented as the mean \pm standard deviation and dichotomous data as a percentage. Comparisons among three groups were performed using one-way analysis of variance (ANOVA) and non-parametric tests, when appropriate. Correlations between two variables were analyzed using Pearson's test or Spearman's test, when appropriate. To determine independent predictors of the presence of LAA stasis for all patients, logistic regression analysis was performed. Significant variables selected in univariate logistic regression analysis $(\mathrm{p}<0.05)$ were entered into the multivariate analysis. Optimal cut-off values were obtained through receiver operating characteristic (ROC) curves, which combined the higher value of specificity plus sensitivity for the prediction of LAA stasis. A two-sided p-value $<0.05$ was accepted as statistically significant.

All data were analyzed using SPSS version 16.0 (SPSS Inc., Chicago, IL, USA) and MedCalc version 12.5.0.0 (MedCalc Software, Mariakerke, Belgium).

\section{Results}

\section{Patient population}

Four patients were excluded for incomplete $\mathrm{S} / \mathrm{SR}$ analysis due to acquisition with inadequate imaging quality and the final study population consisted of 130 patients. No significant difference in general clinical characteristics was observed among the three groups except heart rate and the percentage of persistent AF $(\mathrm{p}<0.05)$ (Table 1). 


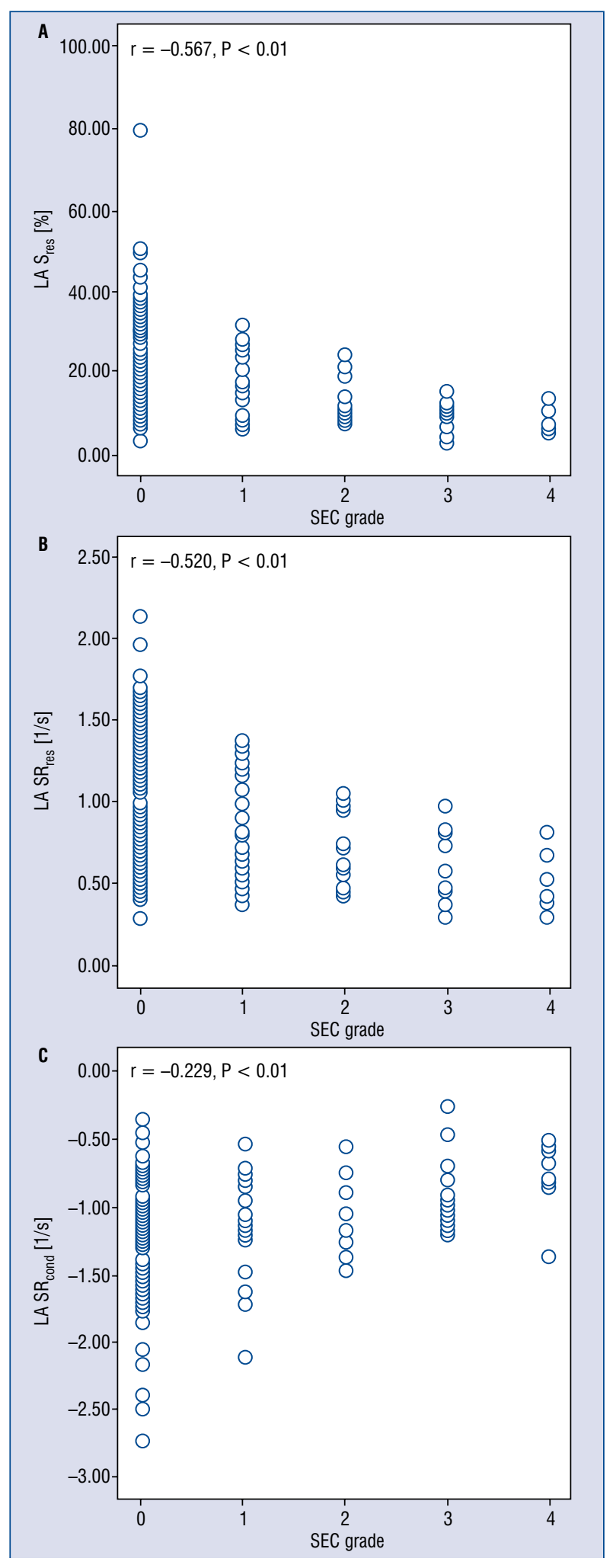

Figure 2. A-C. Relation between left atrial (LA) strain and strain rate (S/SR) with left atrial (LA) spontaneous echo contract (SEC) grade; $S_{\text {res }}$ - global longitudinal strain during atrial reservoir phase; $\mathrm{SR}_{\text {res }}$ - left global longitudinal strain rate during atrial reservoir phase; $\mathrm{SR}_{\text {cond }}$ - global longitudinal strain rate during atrial conduit phase.
In echocardiographic parameters, LAVI and LAA area were larger and LAA function was poorer in group 2 than in group 1 and these differences were much more significant in group $3(\mathrm{p}<0.05)$ (Table 2).

\section{LA S/SR and their relations with SEC grade}

LA $S_{\text {res }}$ and $\mathrm{SR}_{\text {res }}$ were lower in group 2 than in group 1 and were further reduced in group 3 (all $\mathrm{p}<0.01)$. LA SR $\mathrm{SR}_{\text {cond }}$ was decreased in group 3 when compared with that in group 1 and $2(\mathrm{p}<0.05)$, but no significant difference was observed between group 1 and group 2 (Table 3 ). The LA $\mathrm{S}_{\text {res }}$ and $\mathrm{SR}_{\text {res }}$ showed a moderate rank correlation with SEC grade $(r=-0.567$ and -0.520 , respectively; all p < 0.01) (Fig. 2A, B); LA SR cond shown a weak rank correlation with LA SEC grade $(r=0.229$, $\mathrm{p}<0.01$ ) (Fig. 2C).

\section{Relation between LA S/SR and LAA function}

LA $S_{\text {res }}$ and $\mathrm{SR}_{\text {res }}$ were significant correlated with LAA functional parameters, including the LAA $\mathrm{EF}(\mathrm{r}=0.602$ and 0.619 , respectively; $\mathrm{p}<0.01$ ) (Fig. 3A, B) and LAA peak emptying flow velocity $(\mathrm{r}=0.623$ and 0.642 , respectively; $\mathrm{p}<0.01$ )

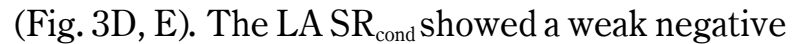
correlation with LAA EF $(r=-0.351, p<0.01)$ (Fig. 3C) and with LAA peak emptying flow velocity $(\mathrm{r}=-0.431, \mathrm{p}<0.01)$ (Fig. 3F).

\section{LA S/SR in prediction of LAA stasis}

Logistic regression analysis was performed to identify independent predictors of the presence of LAA stasis (Table 4). In univariate logistic regression analysis, $\mathrm{LA} \mathrm{S}_{\text {res }}$, $\mathrm{LA} \mathrm{SR}_{\text {res }}$, LA SR $\mathrm{SRn}_{\text {cond }}$ and LAVI were significantly associated with LAA stasis. In multivariate logistic regression analysis, LA $\mathrm{S}_{\text {res }}$ (odds ratio [OR] $0.76,95 \%$ confidence interval [CI] 0.59-0.978; $\mathrm{p}=0.033$ ) and LAVI (OR 1.054, 95\% CI 1.001-1.110; $\mathrm{p}=0.047$ ) were found to be independent predictors of LAA stasis. Besides, LA $\mathrm{S}_{\text {res }}$ is the strongest independent predictor.

The optimal cut-off point of LAVI was recommended as $40 \mathrm{~mL} / \mathrm{m}^{2}$ (area under curve [AUC] value, 0.84 ; sensitivity, $86 \%$; specificity, $68 \%$; negative predictive values, $96 \%$; positive predictive values, $34 \%$ ). And the optimal cut-off point of LA $\mathrm{S}_{\text {res }}$ was recommended as $13 \%$ (AUC value, 0.86; sensitivity, $90 \%$; specificity, $74 \%$; negative predictive values, $98 \%$; positive predictive values, $40 \%$ ), which was better than that of the LAVI in accuracy for predicting LAA stasis (Fig. 4). 


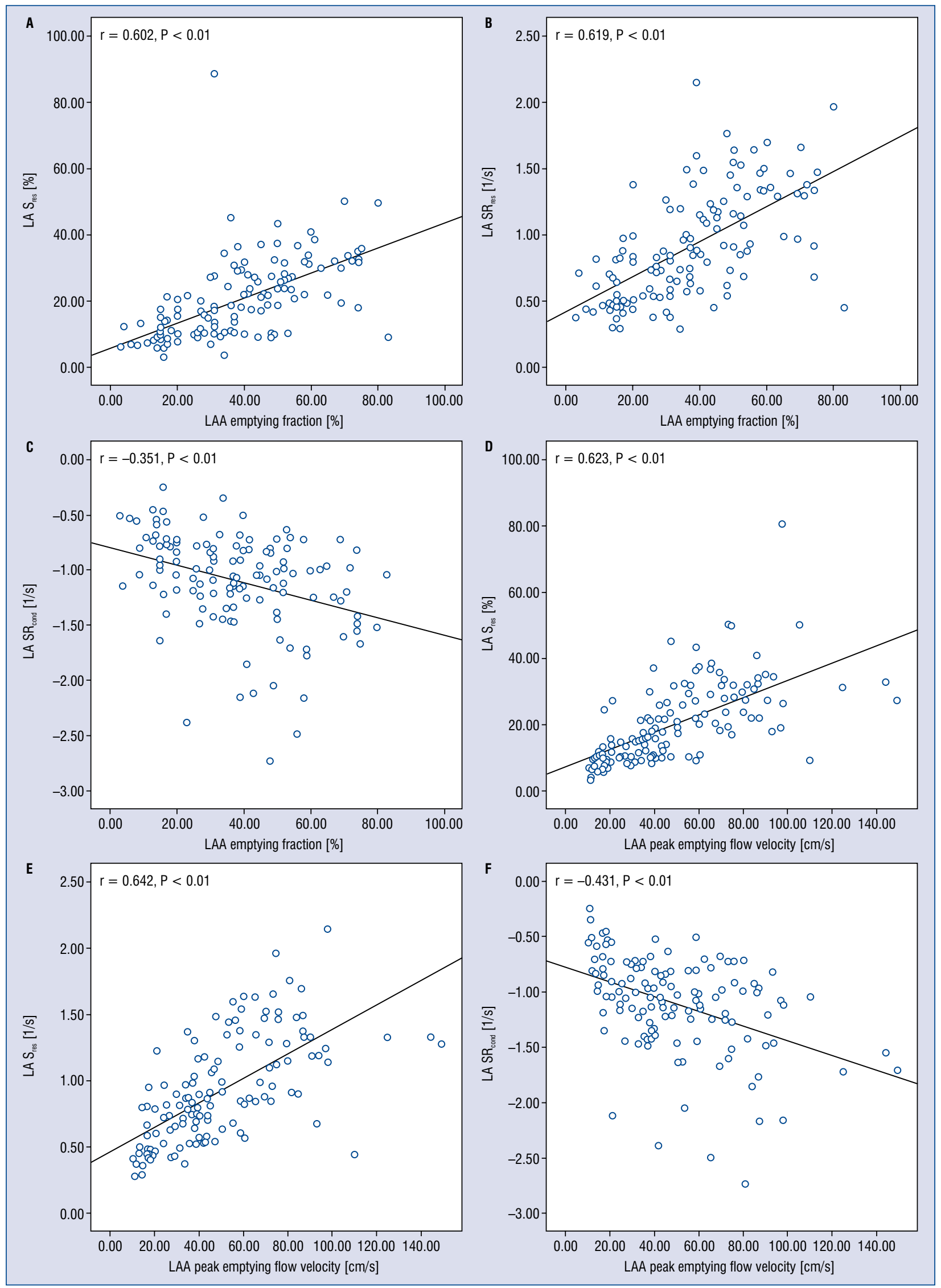

Figure 3. Correlation of left atrial (LA) strain and strain rate (S/SR) with left atrial appendage (LAA) functional parameters; $\mathrm{S}_{\text {res }}$ - global longitudinal strain during atrial reservoir phase; $\mathrm{SR}_{\text {res }}$ - global longitudinal strain rate during atrial reservoir phase; $\mathrm{SR}_{\text {cond }}$ - global longitudinal strain rate during atrial conduit phase. 
Table 4. Univariate and multivariate logistic regression analyses for left atrial appendage stasis.

\begin{tabular}{|c|c|c|c|}
\hline & Odds ratio & $95 \%$ confidence interval & $\mathbf{P}$ \\
\hline \multicolumn{4}{|l|}{ Univariate analyses } \\
\hline LA S $_{\text {res }}$ & 0.760 & $0.658-0.877$ & 0.000 \\
\hline $\mathrm{LA} \mathrm{SR}_{\text {res }}$ & 0.007 & $0.001-0.081$ & 0.000 \\
\hline $\mathrm{LA} \mathrm{SR}_{\text {cond }}$ & 13.483 & $2.463-73.803$ & 0.003 \\
\hline LAVI & 1.106 & $1.056-1.160$ & 0.000 \\
\hline $\mathrm{CHA}_{2} \mathrm{DS}_{2}$-VASc & 1.144 & $0.782-1.673$ & 0.490 \\
\hline History of coronary artery disease & 2.708 & $0.335-21.902$ & 0.350 \\
\hline Hypertension & 1.354 & $0.506-3.624$ & 0.546 \\
\hline Persistent AF & 1.345 & $0.528-3.434$ & 0.533 \\
\hline $\mathrm{E} / \mathrm{e}^{\prime}$ ratio & 1.111 & $0.969-1.274$ & 0.131 \\
\hline Heart rate & 1.017 & $0.997-1.037$ & 0.096 \\
\hline \multicolumn{4}{|l|}{ Multivariate analyses } \\
\hline LA S $_{\text {res }}$ & 0.760 & $0.590-0.978$ & 0.033 \\
\hline $\mathrm{LA} \mathrm{SR}_{\text {res }}$ & 2.760 & $0.022-344.672$ & 0.680 \\
\hline $\mathrm{LA} \mathrm{SR}_{\text {cond }}$ & 0.716 & $0.066-7.776$ & 0.784 \\
\hline LAVI & 1.054 & $1.001-1.110$ & 0.047 \\
\hline
\end{tabular}

AF - atrial fibrillation; E - early diastolic mitral inflow; $\mathrm{e}^{\prime}$ - early diastolic mitral annular tissue velocity; LA — left atrial; LAVI — left atrial

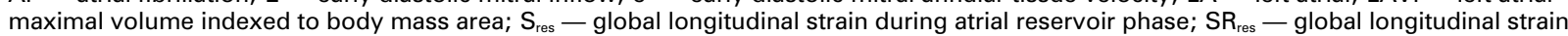
rate during atrial reservoir phase; $\mathrm{SR}_{\text {cond }}$ - global longitudinal strain rate during atrial conduit phase

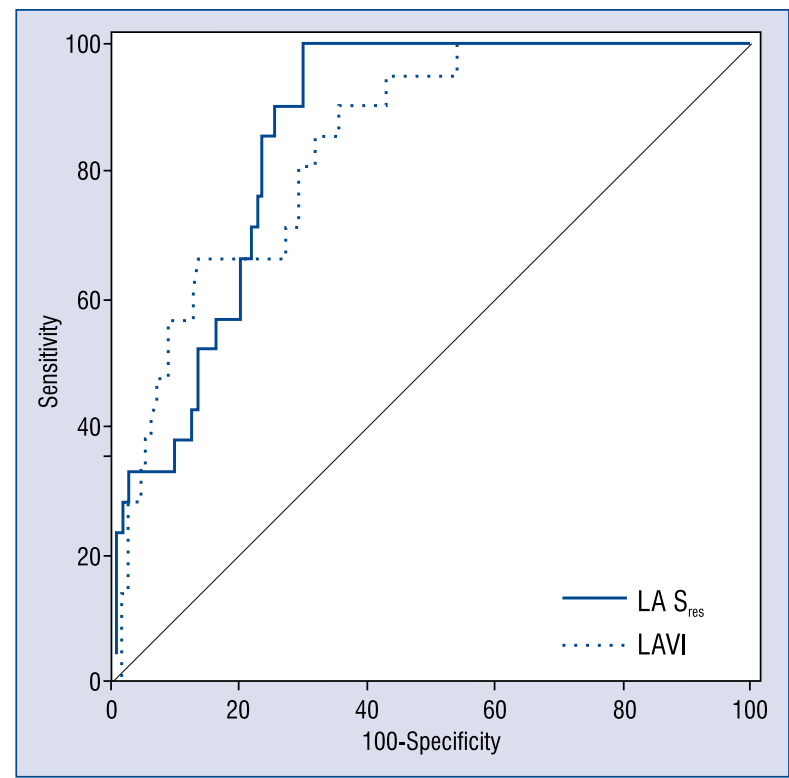

Figure 4. Receiver operating characteristic curves of LA $\mathrm{S}_{\text {res }}$ and LAVI for predicting left atrial appendage stasis; LA - left atrial; LAVI - left atrial maximal volume indexed to body mass area; $\mathrm{S}_{\text {res }}$ - global longitudinal strain during atrial reservoir phase.

\section{Discussion}

This study was attempted to find a new noninvasive and applicable image modality for screening
LAA stasis. The relationships between LA S/SR on TTE, especially during the atrial reservoir phase, and LAA stasis were demonstrated in this study. Besides, the multivariate logistic regression analysis showed that LA $\mathrm{S}_{\text {res }}$ was the strongest independent predictor of the presence of LAA stasis.

Conventional 2D TTE has a low sensitivity of only 3-19\% for the detection of thrombi in LA and especially in LAA [10]. When the formed thrombi are absent, a dense SEC has been demonstrated to be a strong predictor of ischemic stroke [11]. Although TEE detects LA or LAA thrombi with a sensitivity and specificity of 95-100\%, it may not be performed arbitrarily as it is a semi-invasive procedure [12]. Therefore, it would be helpful for guiding TEE application if there are some other noninvasive imaging modalities for predicting LAA stasis in contemporary clinical practice.

Several studies were attempted to resolve the problem, such as that performed by Tamura et al. [13] and Uretsky et al. [14]. They suggested that Doppler tissue velocity of the LAA tip measured from the TTE was an independent predictor of LAA thrombus formation and may be useful for risk stratification of AF. However, the LAA is generally difficult to be clearly and totally visualized on TTE, which results in a challenge to acquire satisfactory LAA velocity indices by TTE. The feasibility and reliability of LA STE-S/SR has been demonstrated 
to have adequate tracking quality in $93 \%$ of patients with $\mathrm{AF}$ with inter- and intra-observer variability ranging between $2.9 \%$ and $10.5 \%$ [15]. It took about 2-3 min to do LA S/SR assessment for each patient after practice. So the LA S/SR analyses would be more clinically practicable. It will provide incremental information and could be integrated in the assessment and the risk stratification of $\mathrm{AF}$ patients.

It has been reported that decreased LAA EF and reduced LAA emptying velocity measured by TEE correlate strongly with the presence of SEC and thrombus in LA or LAA [16, 17]. The correlations between LA S/SR with LAA function parameters, and SEC grade, show the potential application of LA S/SR in predicting LAA stasis. Compared with the modest correlations between LA S/SR in reservoir phase and those parameters above, LA SR $\mathrm{S}_{\text {cond }}$ only had a weak negative correlation with them. A potential reason might be that the decreased S/SR during the reservoir phase has been closely correlated with structural and electrophysiological atrial remodeling during $\mathrm{AF}$, including LA dilatation and increased interstitial fibrosis as documented by magnetic resonance imaging [18]. But LA $\mathrm{SR}_{\text {cond }}$ is also subject to $\mathrm{LV}$ pressure, besides reflecting LA intrinsic deformation properties.

In this study, there were significant differences concerning heart rate and the incidence of persisting $\mathrm{AF}$ among three groups. The incidence of persistent $\mathrm{AF}$ in patients with SEC or thrombi was higher than in patients without such changes. This phenomenon was also reported by Steinberg et al. [19]. The mechanism may be multifactorial, in which long period of LAA/LA stunning and changes in the LA wall structure are included. LA structural remodeling and mechanic functional impairment induced by persistent AF may be expressed by increased LAVI and decreased LA S. According to the result of univariate analysis, heart rate, persisting $\mathrm{AF}$, as well as $\mathrm{CHA}_{2} \mathrm{DS}_{2}$-VASc, coronary artery disease, hypertension, and $\mathrm{E} / \mathrm{e}$ ' ratio, were not significant in predicting LAA stasis.

$\mathrm{LA}_{\mathrm{res}}$ was found to be the strongest independent predictor of LAA stasis in multivariate analysis, followed by LAVI. LA SR $\mathrm{res}_{\text {res }}$ and $\mathrm{LA} \mathrm{SR}_{\text {cond, }}$, which were found significant in univariate analysis, no longer maintained their significance in multivariate analysis. We thought it should be rational for an association of LA strain rather than strain rate with LA stasis, since probably the overall amount of deformation (lengthening or shortening) may be more strongly related to changes of
LA volume and contribute more to maintaining LA reservoir and emptying function than the speed at which deformation occurs. In our sample, LAVI was also shown to be an independent predictor of LAA stasis, although it was not as strong as LA $\mathrm{S}_{\text {res }}$. The associations between LA size and TEE changes were also described in the previous studies [20]. In this study, the significant difference in LAVI among three groups, which were classified according to SEC grade, were also consistent with these points of view. The recommended cut-off points for LA $S_{\text {res }}$ and LAVI for predicting LAA stasis, which were identified by the constructed ROC curves, were $13 \%$ and $40 \mathrm{~mL} / \mathrm{m}^{2}$, with sensitivity $90 \%$ and $86 \%$, specificity $74 \%$ and $68 \%$, respectively. The AUC value of $\mathrm{LA} \mathrm{S}_{\text {res }}$ was higher than that of LAVI. Consequently, LA S ${ }_{\text {res }}$ would be better than LAVI in accuracy for predicting LAA stasis based on multivariate logistic regression and ROC analysis.

The reason of LA $_{\text {res }}$ being valuable in predicting LAA stasis should be mainly because of the relations between LA function and LAA stasis. LA S can be used to evaluate LA function in a sensitive and accurate manner [21]. The primary mechanism of thrombus formation in LA or LAA is their dysfunction in patients with AF. In healthy individuals, the highly dynamic LA and LAA structure prevents stasis. In case of its dysfunction, SEC and/ or thrombus formation may develop in association with increased stasis [22, 23]. LAA develops as a residue originating from LA at 4 weeks of embryonic development. Although the ultrastructural and physiological characteristics of LAA develop independently from LA in this process, muscle cell structures and the myocardium of both structures are similar [22]. Thus, as a component of LA, LAA functions are thought to be reflected by LA structural and functional parameters. A positive correlation between LA and LAA functions has already been reported in several studies [24]. Meanwhile, the relationship between LA S/SR and LAA functional parameters demonstrated in this study also confirms this point of view.

\section{Limitations of the study}

Firstly, the medication for anticoagulation before $\mathrm{AF}$ ablation depends on the physicians in charge. Warfarin's impact on LAA blood status was debatable [25]. In this study, there was no significant difference in anticoagulation status among three groups. Considering the LAA stasis's definition including SEC $3+$, SEC $4+$, and thrombi in this study, the influence of anticoagulant therapy on 
the results should be able to be ignored. Secondly, this was a relatively small study; further confirmation of our results is needed in larger prospective investigations.

\section{Conclusions}

In conclusion, decreased LA strain in reservoir phase by TTE may be a novel noninvasive index for predicting LAA stasis in patients with NVAF. It may be useful for the detection of LAA thrombus and risk stratification of embolism.

\section{Acknowledgments}

The study was funded (Grant No. 134119a5801) by the Science and Technology Committee of Shanghai Municipal Government, People's Republic of China.

\section{Conflict of interest: None declared}

\section{References}

1. Blackshear JL, Odell JA. Appendage obliteration to reduce stroke in cardiac surgical patients with atrial fibrillation. Ann Thorac Surg. 1996; 61(2): 755-759, doi: 10.1016/0003-4975(95)00887-X, indexed in Pubmed: 8572814.

2. Providência R, Trigo J, Paiva L, et al. The role of echocardiography in thromboembolic risk assessment of patients with nonvalvular atrial fibrillation. J Am Soc Echocardiogr. 2013; 26(8): 801-812, doi: 10.1016/j.echo.2013.05.010, indexed in Pubmed: 23791115.

3. Pollick C, Taylor D. Assessment of left atrial appendage function by transesophageal echocardiography. Implications for the development of thrombus. Circulation. 1991; 84(1): 223-231, indexed in Pubmed: 2060098.

4. Vianna-Pinton R, Moreno CA, Baxter CM, et al. Two-dimensional speckle-tracking echocardiography of the left atrium: feasibility and regional contraction and relaxation differences in normal subjects. J Am Soc Echocardiogr. 2009; 22(3): 299-305, doi: 10.1016/j.echo.2008.12.017, indexed in Pubmed: 19258177.

5. January CT, Wann LS, Alpert JS, et al. 2014 AHA/ACC/HRS guideline for the management of patients with atrial fibrillation: A report of the American College of Cardiology/American Heart Association Task Force on Practice Guidelines and the Heart Rhythm Society. J Am Coll Cardiol. 2014; 64: e1-76.

6. Lang RM, Badano LP, Mor-Avi V, et al. Recommendations for cardiac chamber quantification by echocardiography in adults: an update from the American Society of Echocardiography and the European Association of Cardiovascular Imaging. J Am Soc Echocardiogr. 2015; 28(1): 1-39.e14, doi: 10.1016/j. echo.2014.10.003, indexed in Pubmed: 25559473.

7. Fatkin D, Kelly RP, Feneley MP. Relations between left atrial appendage blood flow velocity, spontaneous echocardiographic contrast and thromboembolic risk in vivo. J Am Coll Cardiol. 1994; 23(4): 961-969, doi: 10.1016/0735-1097(94)90644-0, indexed in Pubmed: 8106703.

8. Troughton RW, Asher CR, Klein AL. The role of echocardiography in atrial fibrillation and cardioversion. Heart. 2003; 89(12): 1447-1454, doi: 10.1136/heart.89.12.1447, indexed in Pubmed: 14617563.

9. Lowe B, Kusunose K, Motoki H, et al. Prognostic Significance of Left Atrial Appendage "Sludge" in Patients with Atrial Fibrillation: A New Transesophageal Echocardiographic Thromboembolic Risk Factor. J Am Soc Echocardiogr. 2014; 27(11): 1176-1183, doi: 10.1016/j.echo.2014.08.016, indexed in Pubmed: 25262162.

10. Omran H, Jung W, Rabahieh R, et al. Imaging of thrombi and assessment of left atrial appendage function: a prospective study comparing transthoracic and transoesophageal echocardiography. Heart. 1999; 81(2): 192-198, doi: 10.1136/hrt.81.2.192, indexed in Pubmed: 9922358

11. Bernhardt P, Schmidt H, Hammerstingl C, et al. Patients at high risk with atrial fibrillation: a prospective and serial follow-up during 12 months with transesophageal echocardiography and cerebral magnetic resonance imaging. J Am Soc Echocardiogr. 2005; 18(9): 919-924, doi: 10.1016/j.echo.2005.01.028, indexed in Pubmed: 16153514.

12. Klein AL, Murray RD, Grimm RA. Role of transesophageal echocardiography-guided cardioversion of patients with atrial fibrillation. J Am Coll Cardiol. 2001; 37(3): 691-704, doi: 10.1016/ s0735-1097(00)01178-5, indexed in Pubmed: 11693739.

13. Tamura $\mathrm{H}$, Watanabe $\mathrm{T}$, Hirono $\mathrm{O}$, et al. Low wall velocity of left atrial appendage measured by trans-thoracic echocardiography predicts thrombus formation caused by atrial appendage dysfunction. J Am Soc Echocardiogr. 2010; 23(5): 545-552.e1, doi: 10.1016/j.echo.2010.02.006, indexed in Pubmed: 20359865.

14. Uretsky S, Shah A, Bangalore S, et al. Assessment of left atrial appendage function with transthoracic tissue Doppler echocardiography. Eur J Echocardiogr. 2009; 10(3): 363-371, doi: 10.1093/ ejechocard/jen339, indexed in Pubmed: 19193710.

15. Motoki H, Dahiya A, Bhargava M, et al. Assessment of left atrial mechanics in patients with atrial fibrillation: comparison between two-dimensional speckle-based strain and velocity vector imaging. J Am Soc Echocardiogr. 2012; 25(4): 428-435, doi: 10.1016/j. echo.2011.12.020, indexed in Pubmed: 22265458.

16. Shimizu T, Takada T, Shimode A, et al. Association between paroxysmal atrial fibrillation and the left atrial appendage ejection fraction during sinus rhythm in the acute stage of stroke: a transesophageal echocardiographic study. J Stroke Cerebrovasc Dis. 2013; 22(8): 1370-1376, doi: 10.1016/j.jstrokecerebrovasdis.2013.03.020, indexed in Pubmed: 23608370.

17. Transesophageal echocardiographic correlates of thromboembolism in high-risk patients with nonvalvular atrial fibrillation. The Stroke Prevention in Atrial Fibrillation Investigators Committee on Echocardiography. Ann Intern Med. 1998; 128(8): 639-647, doi: 10.7326/0003-4819-128-8-199804150-00005, indexed in Pubmed: 9537937.

18. Kuppahally SS, Akoum N, Burgon NS, et al. Left atrial strain and strain rate in patients with paroxysmal and persistent atrial fibrillation: relationship to left atrial structural remodeling detected by delayed-enhancement MRI. Circ Cardiovasc Imaging. 2010; 3(3): 231-239, doi: 10.1161/CIRCIMAGING.109.865683, indexed in Pubmed: 20133512.

19. Steinberg BA, Hellkamp AS, Lokhnygina Y, et al. ROCKET-AF Steering Committee and Investigators. Higher risk of death and stroke in patients with persistent vs. paroxysmal atrial fibrillation: results from the ROCKET-AF Trial. Eur Heart J. 2015; 36(5): 288-296, doi: 10.1093/eurheartj/ehu359, indexed in Pubmed: 25209598.

20. Providência R, Botelho A, Trigo J, et al. Possible refinement of clinical thromboembolism assessment in patients with atria fibrillation using echocardiographic parameters. Europace. 2012; 14(1): 36-45, doi: 10.1093/europace/eur272, indexed in Pubmed: 21868410.

21. Saraiva RM, Demirkol S, Buakhamsri A, et al. Left atrial strain measured by two-dimensional speckle tracking represents a new tool to evaluate left atrial function. J Am Soc Echocardiogr. 2010; 23(2): 172-180, doi: 10.1016/j.echo.2009.11.003, indexed in Pubmed: 20152699.

22. Al-Saady NM, Obel OA, Camm AJ. Left atrial appendage: structure, function, and role in thromboembolism. Heart. 1999; 82(5): 547-554, doi: 10.1136/hrt.82.5.547, indexed in Pubmed: 10525506.

23. Wolf PA, Abbott RD, Kannel WB. Atrial fibrillation as an independent risk factor for stroke: the Framingham Study. Stroke. 1991; 22(8): 983-988, doi: 10.1161/01.str.22.8.983, indexed in Pubmed: 1866765.

24. Kaya EB, Tokgözoglu L, Aytemir K, et al. Atrial myocardial deformation properties are temporarily reduced after cardioversion for atrial fibrillation and correlate well with left atrial appendage function. Eur J Echocardiogr. 2008; 9(4): 472-477, doi: 10.1016/j. euje.2007.07.003, indexed in Pubmed: 17826354.

25. Tsai LM, Chen JH, Lin LJ, et al. Natural history of left atrial spontaneous echo contrast in nonrheumatic atrial fibrillation. Am J Cardiol. 1997; 80(7): 897-900, indexed in Pubmed: 9382005. 